\title{
Is a combined therapy more effective than either CBT or SSRI alone? Results of a multicenter trial on panic disorder with or without agoraphobia
}

van Apeldoorn FJ, van Hout WJPJ, Mersch PPA, Huisman M, Slaap BR, Hale WW III, Visser S, van Dyck R, den Boer JA. Is a combined therapy more effective than either CBT or SSRI alone? Results of a multicenter trial on panic disorder with or without agoraphobia.

Objective: To establish whether the combination of cognitivebehavioral therapy (CBT) and pharmacotherapy (SSRI) was more effective in treating panic disorder (PD) than either CBT or SSRI alone, and to evaluate any differential effects between the monotreatments.

Method: Patients with PD $(n=150)$ with or without agoraphobia received CBT, SSRI or CBT + SSRI. Outcome was assessed after 9 months, before medication taper.

Results: CBT + SSRI was clearly superior to CBT in both completer and intent-to-treat analysis (ITT). Completer analysis revealed superiority of CBT + SSRI over SSRI on three measures and no differences between CBT and SSRI. ITT analysis revealed superiority of SSRI over CBT on four measures and no differences between $\mathrm{CBT}+\mathrm{SSRI}$ and SSRI.

Conclusion: Both the mono-treatments (CBT and SSRI) and the combined treatment $(\mathrm{CBT}+\mathrm{SSRI})$ proved to be effective treatments for PD. At post-test, CBT + SSRI was clearly superior to CBT, but differences between CBT + SSRI and SSRI, and between SSRI and CBT, were small.

\section{F. J. van Apeldoorn', W. J. P. J. van Hout', P. P. A. Mersch ${ }^{1}$, M. Huisman ${ }^{2}$, B. R. Slaap ${ }^{3}$, W. W. Hale III ${ }^{4}$, S. Visser ${ }^{5}$, R. van Dyck ${ }^{5}$, J. A. den Boer ${ }^{1}$}

${ }^{1}$ University Medical Center Groningen, ${ }^{2}$ University of Groningen, ${ }^{3}$ Global Clinical Development, N.V. Organon, Oss, ${ }^{4}$ Utrecht University, Research Center Adolescent Development and ${ }^{5} \mathrm{VU}$ University Medical Center, Amsterdam, the Netherlands

Key words: panic disorder; agoraphobia; randomized controlled trials; cognitive-behavioral therapy; drug treatment

Franske $\mathrm{J}$ van Apeldoorn (MSc), University Medical Center Groningen, P0 Box 30.001, 9700 RB, Groningen, the Netherlands.

E-mail: f.j.van.apeldoorn@psy.umcg.nl

Accepted for publication December 19, 2007

\section{Significant outcomes}

- The additive value of a CBT package (consisting of interoceptive exposure, cognitive therapy and exposure in vivo) to an SSRI-only treatment was limited when evaluated after 9 months of treatment, before tapering medication.

- CBT treatment was found to be transportable from academic to non-academic clinical sites. Patients were treated in both research and non-research settings and no site-effects (regarding baseline characteristics, dropout rates or treatment outcome) were detected.

\section{Limitations}

- Baseline data was not collected from patients who lost eligibility prior to starting treatment. These patients could therefore not be included in the ITT analysis.

- To ensure external validity, patients were allowed (minimal) additional benzodiazepine use, and formal treatment integrity checks were not applied. 


\section{Introduction}

The lifetime prevalence rate for panic disorder (PD) without agoraphobia (AG) is estimated at $3.7 \%$, and for PD with $A G$ at $1.1 \%$ (1). In clinical samples, the majority of the patients with PD also suffer from AG. There is a wealth of data supporting the efficacy of both cognitive-behavioral therapy (CBT) and pharmacotherapy using serotonin selective reuptake inhibitors (SSRI) in the treatment of PD (2-5).

The successes of both pharmacotherapy and psychological treatments have led to the hope that the combination of these modalities might further increase efficacy. The added value of a combined treatment may stem from an attenuating effect of the medication on anxiety which in turn might facilitate the exposure component in CBT (6). It is suggested, however, that this attenuating effect lasts only as long as medication is continued (7). If this is true, the superiority of the combined treatment is most evident when treatment effect is assessed before medication taper.

Several studies investigated the efficacy of the combination of antidepressants with CBT (8-17). However, only two studies compared both monotreatments (CBT-only and antidepressants-only) with a combination of the two $(8,16)$.

Sharp et al. (16) compared five treatments: CBT + fluvoxamine, fluvoxamine, CBT, CBT + placebo and placebo $(n=190)$. After 12 weeks, before medication taper, the combination of CBT + fluvoxamine was found to be equally effective as the other active treatments. A similar design was employed in the study by Barlow et al. (8). Patients $(n=312)$ were randomly assigned to receive $\mathrm{CBT}+$ imipramine, imipramine, CBT, CBT + placebo and placebo. After 9 months, before medication taper, the combination of CBT and imipramine was superior to all other treatments on one of two main outcome measures, a clinician-rated scale of PD severity.

To our knowledge, head-to-head comparisons between CBT and antidepressants were made in five studies $(8,16,18-20)$. Before medication taper, Clark et al. (20) found imipramine and cognitive therapy (CT) to be equally effective, while Bakker et al. (18) and Black et al. (19) both found an SSRI treatment to be superior to CT. In the Barlow et al. and Sharp et al. studies $(8,16)$, no significant differences were detected between CBT-only and pharmacotherapy-only treatments.
In sum, some evidence favoring the combined treatment over mono-treatments at treatment endpoint was established in one study with imipramine (8), while no add-on effect of the combined treatment was found in another study with fluvoxamine (16). Further, studies show either no differences between CBT and pharmacotherapy, or results favor the SSRI treatment.

When reviewing the data, two related issues must be taken into account. First, the delivered CBT ingredients differ substantially which makes it difficult to compare overall results. Although consensus has not been reached regarding which CBT components are essential and which components might be redundant in the treatment of PD with and PD without AG, (in vivo) exposure is generally considered to be superior to $\mathrm{CT}$ in treating AG (21). In both studies that yielded results favoring SSRI over CT, exposure techniques were not applied notwithstanding the fact that moderate to severe agoraphobics participated in these studies $(18,19)$. Second, although all studies included patients with PD, they differed on the inclusion of agoraphobics. For instance, Barlow et al. (8) included only patients with no or mild AG. Accordingly, methodological differences among studies preclude drawing unambiguous conclusions on outcome.

As a contribution to the quest for the best possible treatment for patients suffering from PD with or without $A G$, this study evaluated three treatments: CBT, SSRI and the combination of the two (CBT + SSRI). Because previous efficacy studies have established the superiority of the treatments under scrutiny over placebo $(3,4)$, a placebo group was not included. Our goal was to establish the effectiveness of treatment for PD in daily clinical practice and to ensure external validity, treatments were delivered at both research and non-research sites, within a naturalistic context. It was expected that any benefits of the combined treatment would be most obvious before medication taper. Therefore, in line with the Barlow et al. study (8), post-test was scheduled after 9 months of treatment so that patients had ample time to benefit from treatment. Medication taper started after post-test was conducted.

Aims of the study

The objective was to establish whether CBT + SSRI was indeed more effective than either CBT or SSRI alone, and to evaluate any differential effects between the mono-treatments. 


\section{Material and methods}

Study design

This study was approved by the medical ethics committee of the University Medical Center in Groningen and subsequently by institutional review boards at each site. Patients were treated in 11 treatment facilities located throughout the Netherlands. Three kinds of sites participated: i) university training and research centers $(n=2)$; ii) university research clinics $(n=2)$; and iii) regular mental health clinics $(n=7)$. The sites were chosen so as to ensure on the one hand the naturalistic nature of the study and on the other hand to allow for control on external validity. Written informed consent was obtained prior to randomization and after a full explanation of procedures. Patients received either CBT, SSRI or CBT + SSRI. Post-test was completed after 9 months before medication taper; i.e. after 18 CBT sessions and/or nine SSRI sessions.

\section{Randomization}

Randomization was stratified by site. For each site, an envelope containing a number of raffle tickets (CBT, SSRI or CBT + SSRI) was present. The number of raffle tickets in the envelope was based on the number of patients the particular site expected to recruit. After drawing lots, the raffle tickets were not placed back in the envelope. When a patient met study criteria, local coordinators of the participating centers contacted the third or first author who performed the drawing at the University Medical Center in Groningen over phone or e-mail. Beforehand, no information about the patient was interchanged.

\section{Study participants}

Regular patients seeking care at the participating treatment centers and meeting the study criteria were asked to participate in the study. Patients were also recruited through media advertisements and flyers which were distributed in general practitioner offices. Patients were enrolled between April 2001 and September 2003. Screening consisted of a structured interview, the MINI (22), checking DSM-IV criteria for Axis I disorders. Randomized patients suffered from a primary diagnosis of PD with or without AG. Once included, AG level was assessed by the first author based on chart review and the structured interview. Patients were classified as not suffering from AG, or suffering from mild, moderate or severe AG following guidelines set forth by the DSM-III-R.
Inclusion was restricted to patients between 18 and 65 years of age. Patients who were pregnant, lactating, suicidal, psychotic or severely depressed were ineligible to participate in the study. Further exclusion criteria comprised contra-indications to either treatment or a concurrent competing treatment. Patients were not allowed to use psychotropic drugs except small doses of benzodiazepines (maximum the equivalent of $20 \mathrm{mg}$ of oxazepam per day).

\section{Treatment conditions}

Each of the three treatments was delivered in every treatment center. Before the start of the study, coworkers of all participating treatment centers assembled to discuss the treatment modalities and to integrate existing views. Because the study was designed to follow common practice in the treatment of PD, the treatment manuals were based on the outcomes of these gatherings to satisfy as closely as possible 'care as usual' requirements. The manual-based treatments are summarized below.

$C B T$. The CBT protocol is based on the work of Clark (23) and Craske and Barlow (24). Patients in the CBT group received up to $18 \mathrm{CBT}$ sessions each lasting approximately $50 \mathrm{~min}$. To prevent return of fear, interval between sessions were extended in the course of treatment (from once a week to twice a week, and from session 16 onward with 5 week intermissions) (25). During the first session, the treatment rationale was provided which was based on the cognitive model of panic as developed by Clark (23). In the second session, interoceptive exposure was introduced and exercises were performed (throughout sessions two to six) to provoke relevant bodily sensations. By performing those exercises patients were taught that bodily sensations can indeed be provoked, that these sensations spontaneously subside, and that these sensations are not dangerous and are not followed by any harmful consequences. From session 6 onward, patients received CT. During $\mathrm{CT}$, patients were first taught about the role of thoughts in generating emotions. Detailed discussion of emotions and associated cognitions led to the identification of specific beliefs, appraisals and assumptions. Patients were encouraged to examine the validity of their cognitions by considering all the available evidence and actively collecting new evidence. Both automatic appraisals (such as if I panic, I will faint') and core-level beliefs or schemata (such as 'I am weak') were examined in this manner. Based on this hypothesis testing, alternative hypotheses were generated that were 
evidence based. In the 10th session, exposure in vivo was introduced. When starting exposure in vivo, an individualized fear hierarchy was constructed. In between sessions, patients conducted self-guided exposure in vivo. Each exposure assignment was carefully designed and written down jointly by a therapist and the patient. Patients were instructed to stay in the feared situation until their anxiety level had dropped significantly. Safety-seeking behaviors were prohibited during the exposure exercises.

From session 10 onwards, both CT and exposure in vivo were offered. The emphasis on one of the two was left to the clinical judgment of the therapist. Homework assignments were given throughout the treatment and were thoroughly discussed at the beginning of each session. Each new treatment component was introduced with a separate treatment rationale. These rationales were handed out to patients on paper so they could read them at home. A treatment manual, which contained detailed information about each session, was provided to all CBT therapists. Following each treatment session, all therapists (including pharmacotherapists) completed a detailed form regarding the content of that session. These forms were evaluated by the research team to check treatment adherence.

SSRI. Patients receiving an SSRI visited their therapist nine times, with weekly sessions during the first month and the remaining sessions distributed evenly over the treatment period. Each visit lasted approximately $20 \mathrm{~min}$. SSRI prescriptions were in conformance with the pharmacotherapeutical guidelines as formulated by the Dutch Psychiatry Association (26). Pharmacotherapists could choose between five SSRIs currently prescribed in the Netherlands: fluvoxamine, fluoxetine, paroxetine, sertraline and citalopram. During the first SSRI session, patients received some general information on the role of serotonergic pathways in the brain involved in anxiety disorders and the working of SSRIs in PD. Patients were administered a minimum dosage which was titrated upwards up to the effective range in the first month, and adjusted according to clinical response and tolerability. Pharmacotherapists were instructed to withhold from therapeutical interventions to avoid hidden exposure. Initiatives for exposing oneself to avoided situations were left to the patient.

$C B T+S S R I$. This treatment was administered according to the CBT and SSRI manuals. The two treatments started simultaneously and were delivered parallel. The CBT was delivered by the
CBT therapist and the SSRI treatment was delivered by the pharmacotherapist.

\section{Therapists}

In the two university training and research centers, CBT was delivered by master-level student therapists who underwent extensive training and who were closely supervised during weekly gatherings. In the remaining treatment centers, CBT was performed by experienced clinical psychologists. All therapists received ongoing supervision on site. The SSRI treatment was delivered by experienced psychiatrists, psychiatrists in training or trained physicians. The research team, situated at the University Medical Center in Groningen, was always available for any questions regarding the treatment (manual) or study design.

Assessment

In this study, we will report on six outcome measures: four continuous measures (regarding avoidance, anxiety, depression and general psychopathology) and two categorical measures (responder status and panic-free status).

Patients were defined panic free when they reported no panic attacks in a panic log during a 2-week period after 9 months of treatment (posttest). Also at post-test, PD severity and degree of improvement were evaluated by both the patient and an independent rater using the patient global evaluation (PGE), and the Clinical Global Improvement scale (CGI) respectively (27). On the improvement scales, a score of 1,2 or 3 (very much improved to improved) was needed to meet responder criteria. On the severity scales, a score of 1 or 2 (no complaints or only mild complaints) was needed. Patients were classified as responders when they met these criteria for at least three of four PGE/CGI scales (PGE Improvement, PGE Severity, CGI Improvement and CGI Severity).

The Hamilton Anxiety Rating Scale (HAM-A) (28) and the Hamilton Depression Rating Scale (HAM-D) (29) were administered by trained research assistants. Two self-report questionnaires yielded information regarding agoraphobic avoidance (the five-item agoraphobia subscale of the Fear Questionnaire (FQ-AG) (30), and regarding general psychopathology (the total score on the Symptoms Checklist (SCL-90) $(31,32)$.

\section{Statistical analyses}

Pretest differences between randomized groups were analyzed using univariate analyses of variance 
(ANOVAS) or non-parametric equivalents if called for. Chi-squared analyses, Fisher's exact tests and an analysis of covariance were used to detect possible site differences. Time effects per treatment were analyzed with $t$-tests for dependent samples. Differential treatment effects were analyzed twice: for the completer $(n=100)$ and for the intent-totreat (ITT; $n=145$ ) samples. In the ITT analysis, pretest scores for dropout patients were carried forward to post-test as an assumption of nonresponse or return to pretest level. Note that because no pretest scores for responder status were present, only completer results are available for this particular outcome measure.

Chi-squared analyses were used to investigate overall differences in responder- and panic-free rates. Pair-wise differences between proportions were evaluated using the Wilson 95\% confidence interval (CI) around this difference (33). Analyses of covariance (ANCOVAS), using the pretest score as covariate, were used for all continuous measures. Contrasts (non-orthogonal) were computed to evaluate pair-wise differences among the adjusted mean values. For the multiple comparisons, alpha levels were corrected according to the BonferroniHolm procedure (34). When CBT + SSRI was compared with either mono-treatment, the onetailed $P$-value was used. Post hoc effect sizes (ES) (indexed using Pearson's correlation coefficient $r=\sqrt{ } t^{2} / \mathrm{t}^{2}+$ d.f. which ranges from 0 to 1$)$ were calculated for both the within-group pre- to posttest changes and all between-group comparisons. According to suggestions made by Cohen (35), an ES of \pm 0.10 constitutes a small effect, an ES of \pm 0.30 constitutes a medium effect and an ES of \pm 0.50 constitutes a large effect.

Unless stated differently, tests were two-tailed and alpha was set at 0.05 .

\section{Results}

Intake summary and pretest analyses

One hundred and seventy-eight eligible patients were seen for screening (see Fig. 1). Subsequently, 150 patients started with CBT + SSRI $(n=49)$, CBT $(n=53)$, or SSRI $(n=48)$. In total, $54.7 \%$ of the sample were women (see Table 1). Mean age was 37.5 years (range 18-61 years).

Sixty-three patients were treated at the two university training and research centers, 42 at the two university research clinics and 45 patients at the seven regular mental health clinics. When analyzing pretest scores, a significant difference was detected on the CGI $(F=5.7$; d.f. $=2$; $P=0.004)$ indicating that patients treated at the

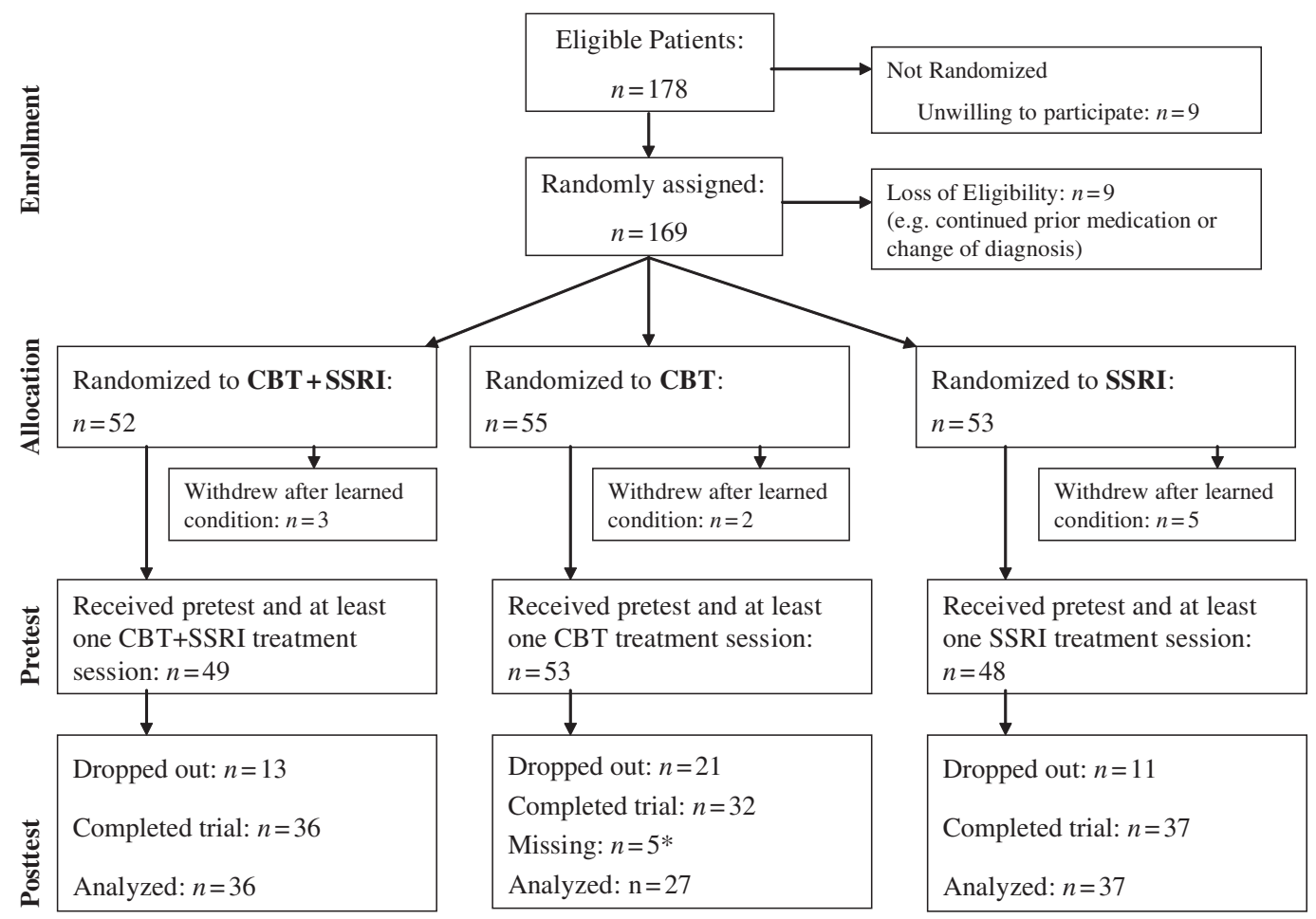

Fig. 1. Flow diagram. CBT, cognitive-behavioral therapy; SSRI, treatment with selective serotonin reuptake inhibitor; CBT + SSRI, combined CBT + SSRI treatment. *No data available: completed CBT before 9 months due to early treatment success. 
Table 1. Pretest characteristics of patients with panic disorder with or without agoraphobia who received CBT, SSRI or CBT + SSRI

\begin{tabular}{|c|c|c|c|c|c|c|c|c|}
\hline \multirow[b]{2}{*}{ Pretest characteristic } & \multicolumn{2}{|c|}{ CBT $(n=53)$} & \multicolumn{2}{|c|}{ SSRI $(n=48)$} & \multicolumn{2}{|c|}{$\begin{array}{c}\text { CBT + SSRI } \\
(n=49)\end{array}$} & \multicolumn{2}{|c|}{$\begin{array}{l}\text { All Patients } \\
(n=150)\end{array}$} \\
\hline & Mean & SD & Mean & SD & Mean & SD & Mean & SD \\
\hline Duration of illness in years & 8.1 & 8.4 & 10.2 & 10.4 & 7.2 & 7.6 & 8.5 & 8.9 \\
\hline Age & 39.4 & 10.2 & 38.5 & 10.5 & 34.4 & 10.6 & 37.5 & 10.6 \\
\hline Number of panic attacks* & 5.44 & 9.57 & 3.62 & 4.50 & 5.08 & 5.75 & 4.74 & 6.99 \\
\hline PGE, severity & 3.45 & 1.08 & 3.53 & 1.06 & 3.51 & 1.12 & 3.47 & 1.07 \\
\hline CGI, severity & 2.94 & 1.00 & 2.90 & 0.84 & 2.76 & 0.94 & 2.89 & 0.95 \\
\hline FO subscale agoraphobia & 18.08 & 11.94 & 17.32 & 12.21 & 20.94 & 10.70 & 18.78 & 11.66 \\
\hline HAM-A & 25.46 & 10.65 & 21.94 & 9.78 & 23.29 & 8.54 & 23.62 & 9.77 \\
\hline HAM-D & 15.21 & 7.30 & 14.23 & 7.47 & 14.61 & 6.51 & 14.70 & 7.07 \\
\hline \multirow[t]{2}{*}{ SCL-90 } & 196.21 & 55.66 & 186.24 & 65.10 & 192.99 & 49.83 & 192.01 & 56.82 \\
\hline & $n$ & $\%$ & $n$ & $\%$ & $n$ & $\%$ & $n$ & $\%$ \\
\hline Female sex & 33 & 62.3 & 26 & 54.2 & 23 & 46.9 & 82 & 54.7 \\
\hline Currently married** & 29 & 54.7 & 33 & 68.8 & 25 & 51.0 & 87 & 58.0 \\
\hline Currently employed & 30 & 56.6 & 31 & 64.6 & 31 & 63.3 & 92 & 61.3 \\
\hline \multicolumn{9}{|l|}{ Level of completed education } \\
\hline Low & 10 & 18.9 & 11 & 24.4 & 11 & 22.4 & 32 & 21.8 \\
\hline Moderate & 28 & 52.8 & 16 & 35.6 & 14 & 28.6 & 58 & 39.5 \\
\hline Above moderate & 6 & 11.3 & 9 & 20.0 & 9 & 18.4 & 24 & 16.3 \\
\hline High & 9 & 17.0 & 9 & 20.0 & 15 & 30.6 & 33 & 22.4 \\
\hline \multicolumn{9}{|l|}{ Level of agoraphobia (AG) } \\
\hline No or mild AG & 26 & 49.1 & 24 & 50 & 23 & 46.9 & 73 & 48.7 \\
\hline Moderate or severe $A G$ & 27 & 50.9 & 24 & 50 & 26 & 53.1 & 77 & 51.3 \\
\hline Benzodiazepine users*** & 18 & 34 & 12 & 25 & 14 & 28.6 & 44 & 29.3 \\
\hline Having received previous CBT treatment & 2 & 3.8 & 3 & 6.4 & 7 & 14.3 & 12 & 8.1 \\
\hline Having received previous SSRI treatment & 17 & 32.1 & 12 & 25.0 & 14 & 28.6 & 43 & 28.7 \\
\hline Comorbid Axis I disorder present & 28 & 52.8 & 20 & 41.7 & 20 & 55.1 & 75 & 50.0 \\
\hline
\end{tabular}

*Mean number of panic attacks during a 2-week pretest period ${ }^{* *}$ or cohabiting with steady partner. ${ }^{* *}$ Benzodiazepine use could vary between very infrequent use to daily use. CBT, cognitive-behavioral therapy; SSRI, treatment with selective serotonin reuptake inhibitor; CBT + SSRI, combined CBT + SSRI treatment; PGE, patient global evaluation; CGI, Clinical Global Impression; FQ, fear questionnaire; HAM-A, Hamilton Anxiety Rating Scale; HAM-D, Hamilton Depression Rating Scale; SCL-90, symptoms checklist. university research clinics had somewhat higher pretest CGI scores than patients treated at the other sites. Also, the level of completed education was lower for patients treated at non-research sites when compared with patients treated at research sites $\left(\chi^{2}=8.8 ;\right.$ d.f. $\left.=3 ; P=0.03\right)$. No other significant site differences were detected at baseline (all $P \geq 0.22$ ).

The classification of AG types (no, mild, moderate and severe) was supported using pretest scores on the FQ-AG. The following mean values were found: no AG: mean FQ-AG 6.3 (SD 6.3), mild AG: mean FQ-AG 13.3 (SD 9.1), moderate AG: mean FQ-AG 22.4 (SD 10.4) and severe AG: mean FQ-AG $31.1(\mathrm{SD} 8.0)(F=29.1$; d.f. $=3$; $P<0.001)$. About half of the patients $(48.7 \%)$ had no or mild AG, whereas $51.3 \%$ suffered from moderate or severe AG. At pretest, patients with moderate or severe AG obtained significantly higher mean values (scored more severe) than patients without or with mild AG on each outcome measure (all $P \leq 0.05$ ).

Patients in the CBT + SSRI group were slightly younger than in the other treatment groups $(F=3.23 ;$ d.f. $=2 ; P=0.04)$ with mean age ranging from 34.4 (CBT + SSRI) to 39.4 (CBT). No other significant differences regarding patient characteristics among the three randomized treatment groups were found (all $P \geq 0.18$ ). In addition, analyses performed on each outcome measure yielded no significant differences between treatment groups at pretest (all $P \geq 0.27$ ).

Attrition. A total of 45 patients $(30 \%$ of 150 patients who had started treatment) dropped out of treatment. There was no significant difference in patient dropout rate between treatment groups, although more patients dropped out of CBT (39.6\%: $n=21)$ when compared with CBT + SSRI $(26.5 \%: \quad n=13)$ and SSRI $(22.9 \%: \quad n=11) \quad\left(\chi^{2}=3.76 ; \quad\right.$ d.f. $=2$; $P=0.15)$. When compared with completers, significantly more patients who subsequently dropped out had previously used an SSRI $(P=0.02$, Fisher's exact test). No other significant differences between completers and dropouts on outcome variables or regarding patient characteristics at pretest were found (all $P \geq 0.07$ ).

Reasons for dropout included side effects of medication $[n=11 \quad(24.4 \%)$, including four 
CBT + SSRI and seven SSRI patients], life events not related to treatment $[n=3(6.7 \%)$, all three CBT patients], non-compliance $[n=10(22.2 \%)$, including four CBT + SSRI, five CBT and one SSRI patients], needing other treatment $[n=6$ $(13.3 \%)$, including three CBT + SSRI, two CBT and one SSRI patients], loosing motivation because of good results $[n=1(2.2 \%)$, CBT patient], not satisfied with obtained results $[n=5$ $(11.1 \%)$, including one CBT + SSRI and four CBT patients], using medication outside protocol $[n=3(6.7 \%)$, all three CBT patients $]$ and unknown reasons $[n=6(13.3 \%)$, including one CBT + SSRI, three CBT and two SSRI patients].

Site differences

To detect possible site differences, several analyses were performed. First, dropout rates were compared between the three types of sites (site clusters 1-3, see section: study design) and between research and non-research sites (site clusters $1+2$ compared with 3 ) and no significant differences were found ( $P=0.92$ and 0.85 respectively). Second, possible differences in treatment effect between sites were analyzed and no significant differences were found (all $P \geq 0.09$ ). Finally, analyses were repeated for only the patients who had received CBT because possible site differences were believed to have the greatest impact on this group of patients. Next to comparing the three sites and comparing research and non-research sites, we also checked for differences between sites using student therapists (site cluster 1) and sites not using student therapists (site clusters 2 and 3 ) to see whether therapist level of experience was a factor of importance. For CBT patients, site differences were thus analyzed in three ways and taken these analyses together, no differences in dropout rate (all $P \geq 0.95$ ) or treatment outcome (all $P \geq 0.09$ ) were detected.

\section{Treatment effects}

Table 2 shows the adjusted post-test scores, CIs and ESs for the continuous outcome measures. The post-test proportions of panic-free patients and responders are depicted in Figs 2 and 3 respectively. Mean within-group ESs are presented in Table 3.

Because patients in the CBT + SSRI group were slightly younger than in the other treatment groups, the effect of age on treatment effect was investigated using covariance analysis. No main effect of age was found on any measure (all $P \geq 0.11)$.

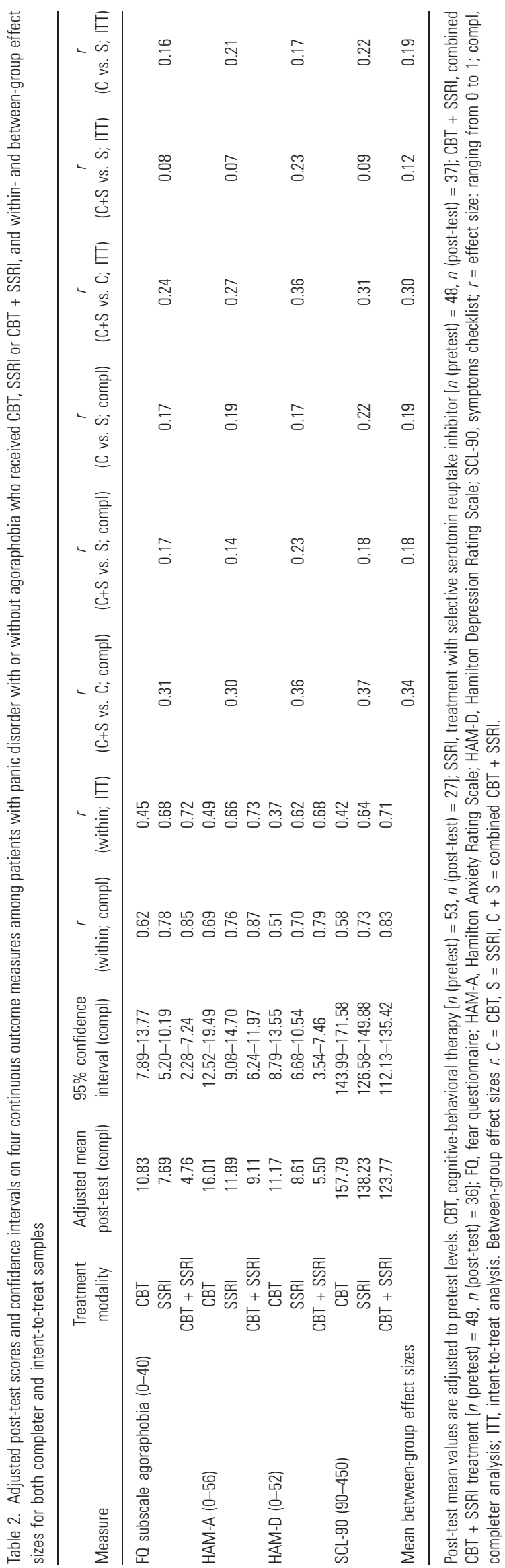




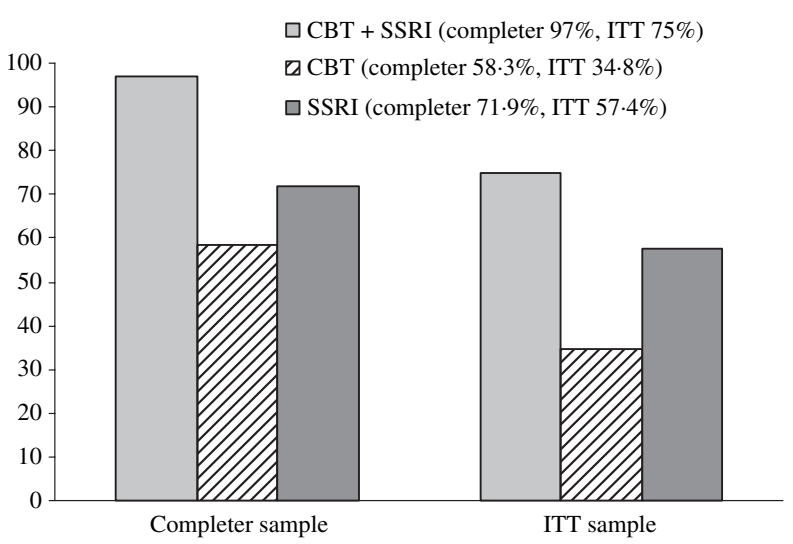

Fig. 2. Proportions of panic-free patients at post-test for both completer and intent-to-treat samples.

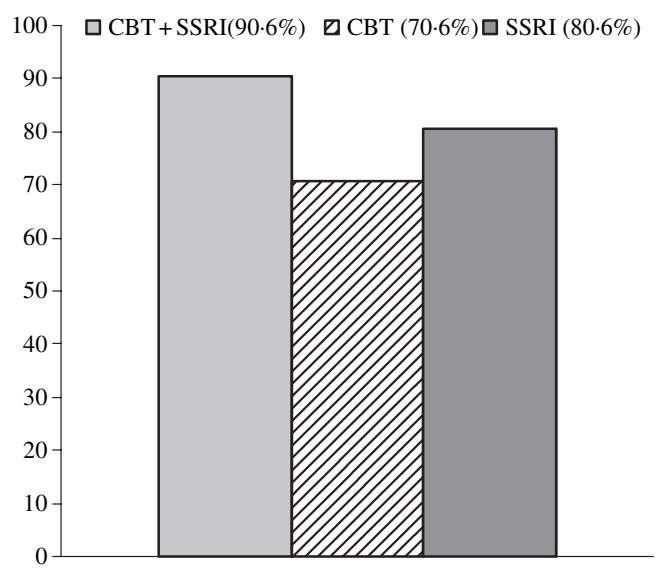

Fig. 3. Proportions of patients meeting responder criteria at post-test for the completer sample.

All patients, regardless of received treatment, showed significant improvements from pre- to post-test on all outcome measures, both in the completer and ITT analyses (all $P \leq 0.01$ ). For the sake of brevity, we will only report the pair wise comparisons. Regarding the ITT analysis, only the differences between the completer and the ITT analysis will be highlighted.

$C B T+S S R I$ vs. CBT. Completer analysis revealed that $\mathrm{CBT}+\mathrm{SSRI}$ was superior to $\mathrm{CBT}$ on the FQ-AG $(F=9.87$; d.f. $=1 ; P=0.001)$, HAM-A $(F=9.17$; d.f. $=1 ; P=0.002)$, SCL-90 $(F=14.0 ; \quad$ d.f. $=1 ; \quad P<0.001), \quad$ HAM-D $(F=13.3 ;$ d.f. $=1 ; P<0.001)$ and regarding the proportion of patients reaching panic-free status $(95 \%$ CI of difference in proportion from 0.17 to 0.58 ). Significance was not reached regarding the proportion of patients reaching responder status $(95 \%$ CI of difference in proportion from -0.36 to 0.13 ). In the ITT analysis, the hypothesized superiority of CBT + SSRI to CBT was
Table 3. Within-group effect sizes for CBT-only, antidepressant-only and the combination of the two for six studies

\begin{tabular}{|c|c|c|c|c|c|c|}
\hline & $\begin{array}{l}\text { Present } \\
\text { study* }\end{array}$ & $\begin{array}{l}\text { Barlow } \\
\text { et al. } \dagger\end{array}$ & $\begin{array}{l}\text { Sharp } \\
\text { et al.\$ }\end{array}$ & $\begin{array}{l}\text { Clark } \\
\text { et al.§ }\end{array}$ & $\begin{array}{l}\text { Bakker } \\
\text { et al. }\end{array}$ & $\begin{array}{l}\text { Black } \\
\text { et al.** }\end{array}$ \\
\hline \multicolumn{7}{|l|}{ Completer } \\
\hline CBT-only & 0.60 & 0.62 & 0.71 & 0.64 & 0.44 & 0.37 \\
\hline Antidepressant-only & 0.74 & 0.72 & 0.51 & 0.53 & $\begin{array}{l}0.67 \text { par., } \\
0.54 \text { clom. }\end{array}$ & 0.63 \\
\hline Combined therapy & 0.84 & 0.80 & 0.69 & - & - & - \\
\hline \multicolumn{7}{|l|}{ Intent-to-treat } \\
\hline CBT-only & 0.43 & 0.41 & - & - & 0.29 & 0.35 \\
\hline Antidepressant-only & 0.65 & 0.45 & - & - & $\begin{array}{l}0.58 \text { par., } \\
0.51 \text { clom. }\end{array}$ & 0.43 \\
\hline Combined therapy & 0.71 & 0.59 & - & - & - & - \\
\hline
\end{tabular}

*Mean effect sizes based on effect sizes in Table 2. CBT: interoceptive exposure, cognitive therapy and exposure in vivo. Antidepressants: fluoxetine, fluvoxamine, citalopram, sertraline or paroxetine.

$\dagger$ Based on mean values and SDs presented in Tables 1 and 2 (8), $d$ was computed for one outcome measure. Subsequently $d$ was converted into $r$. CBT: interoceptive exposure, cognitive restructuring and breathing retraining. Antidepressant: imipramine.

\$Based on the mean values and SDs kindly provided to us by Dr Sharp, Cohen’s $d$ was computed for three outcome measures. The mean $d$ was subsequently converted into r. CBT: cognitive and behavioral panic management techniques including exposure. Antidepressant: fluvoxamine (16). ITT analysis was not performed.

§Based on the mean values and SDs presented in Table 2 (20), Cohen's $d$ was computed for 19 outcome measures. The mean $d$ was subsequently converted into r. CBT: cognitive therapy. Antidepressant: imipramine. ITT analysis was not performed.

- Based on the effect sizes in Table 3 (18), a mean $d$ was computed, subsequently $d$ was converted into $r$. CBT: cognitive therapy. Antidepressants: par. = paroxetine, clom. = clomipramine.

**Based on the mean values and SDs presented in Table 2 (19), Cohen's $d$ was computed for six outcome measures. The mean $d$ was subsequently converted into $r$. CBT: cognitive therapy. Antidepressant: fluvoxamine.

confirmed on all outcome measures (all $P \leq 0.002$, and for proportion of patients reaching panic-free status $95 \%$ CI of difference in proportion from 0.20 to 0.56 ).

$C B T+$ SSRI vs. SSRI. Completer analysis favored CBT + SSRI over SSRI on the HAM-D $(F=5.1 ;$ d.f. $=1 ; P=0.01)$, SCL-90 $(F=3.0$; d.f. $=1 ; P=0.04)$, and regarding the proportion of patients reaching panic-free status $(95 \% \mathrm{CI}$ of difference in proportion from 0.08 to 0.43 ). Significance was not reached regarding the proportion of patients reaching responder status $(95 \%$ CI of difference in proportion from -0.28 to 0.08 ), not on the HAM-A $(F=1.9$; d.f. $=1 ; P=0.08)$, and also not on the FQ-AG $(F=2.7$; d.f. $=1$; $P=0.05)$. In the ITT analysis, no significant differences between CBT + SSRI and SSRI were observed on any measure.

CBT vs. SSRI. Completer analysis showed no significant differences between SSRI and CBT on any measure (all $P \geq 0.03$ ). In the ITT analysis, SSRI was superior to CBT on the HAM-A $(F=6.5 ; \quad$ d.f. $=1 ; \quad P=0.01), \quad$ HAM-D 
$(F=5.1 ;$ d.f. $=1 ; P=0.02)$, SCL-90 $(F=7.0$; d.f. $=1 ; P=0.01)$ and regarding the proportion of patients reaching panic-free status $(95 \%$ CI of difference in proportion from 0.02 to 0.41 ).

In sum, CBT + SSRI was superior to CBT on five outcome measures in both completer and ITT analyses. CBT + SSRI was superior to SSRI on three measures in the completer analysis but not on any measure in the ITT analysis. SSRI did not outperform CBT in the completer analysis but proved superior to CBT on four measures in the ITT analysis. Thus, compared with the completer analysis, CBT performed poorly, whereas SSRI performed better in the ITT analysis.

\section{Discussion}

The present findings corroborate the predicted superiority of CBT + SSRI over CBT. This superiority was demonstrated on all outcome measures in the completer analysis except the categorical outcome measure responder status. The predicted superiority of CBT + SSRI over SSRI was not confirmed. Although the mean post-test scores were consistently lower for CBT + SSRI when compared with SSRI, significance was reached for only three of six measures in the completer and no measures in the ITT analysis suggesting that the added value of CBT to SSRI-only is limited. The small mean between-group ESs (both completer and ITT) for CBT + SSRI vs. SSRI point in the same direction. Note that, to our knowledge, this is the first study on PD to report between-group ES which probably offer the most insight into the magnitude of observed differences between groups.

In the Barlow et al. and Sharp et al. studies (8, 16), no significant differences between CBT-only and antidepressant-only emerged. Present completer analysis revealed no significant differences between the mono-treatments but SSRI was superior to $\mathrm{CBT}$ on four measures in the ITT analysis. This might raise questions regarding the CBT treatment as delivered in this study. To be able to compare the present results to previous findings, (mean) within group ES were computed for relevant studies (see Table 3). In general, substantial differences between completer and ITT ES are observed. This might be explained by diverging attrition rates as an uneven distribution in dropouts between treatments may favor one treatment over another in the ITT analysis. For example, in this study the dropout rates for, respectively, the combined treatment, antidepressant-only treatment and CBT are $26.5 \%, 22.9 \%$ and $39.6 \%$ compared with $38.8 \%, 62.7 \%$ and $37.5 \%$ which we derived from the Barlow et al. study (8). While in this study the highest dropout rate was observed for CBT-only, in the Barlow et al. study, the highest dropout rate was observed for the antidepressant-only treatment. This explains why, in the latter study, the greatest change between completer and ITT ES was observed for the antidepressant-only treatment, while in this study the greatest change between completer and ITT ES was observed for the CBT treatment. Based on the observed ES (CBT this study 0.60 completer, 0.43 ITT, CBT Barlow et al. 0.62 completer, 0.41 ITT) and dropout rates (present $\mathrm{CBT}$ dropout rate $39.6 \%$, CBT dropout rate in the Barlow et al. study $37.5 \%$ ), it seems safe to conclude that CBT in this study did not perform worse compared with previous studies but that the delivered CBT + SSRI and SSRI treatments simply performed better. Please note that the high antidepressant dropout rate in the Barlow et al. study (62.7\% compared with $22.9 \%$ in this study) might be explained by the use of imipramine instead of an SSRI. SSRIs are known for their greater tolerability in comparison with tricyclic antidepressants such as imipramine (36).

Patients with different AG severity levels participated in this study. At pretest, patients with moderate or severe AG obtained significant higher mean values (scored more severe) than patients without or with mild AG on each outcome measure. Post hoc, univariate analyses of variance was applied again but this time on the post-test scores. By that time, except for the FQ-AG, differences between the two AG groups were no longer significant. This might suggest that patients with moderate or severe AG, although still reporting more agoraphobic behavior at post-test, benefit as much from treatment as patients without or with only mild AG. Clearly, the relationship between AG level and treatment outcome warrants further investigation.

Strengths of this study include the fact that both academic and non-academic clinical sites participated. Results show that CBT, SSRI and CBT + SSRI are effective treatments in clinical practice. No site differences were detected and patients improved equally well regardless of whether they were treated at research or nonresearch sites. Criteria for patient selection were liberal, and treatments were delivered according to care as usual. Therefore, the present findings can be considered highly externally valid with respect to type of patients, type of treatments and type of treatment centers.

Establishing the effectiveness of treatment for PD in daily clinical practice was set as the main goal. Therefore, in ensuring external validity, some 
decisions were made which might have affected internal validity. First, although therapists completed a detailed form regarding session content following each treatment session, more formal treatment integrity or treatment fidelity checks (such as audio taping each session or screening blood or urine samples) were not applied because this was found to be logistically impractical and also incompatible with our intention to simulate clinical practice.

Second, patients were allowed comorbid use of benzodiazepines and the effects of benzodiazepines and SSRIs might thus be intertwined. It is, however, unlikely that the present findings can be accounted for with additional benzodiazepine use as pretest benzodiazepine use was evenly distributed across treatments and can therefore not account for the observed post-test differences in outcome. Also, Otto et al. (37) found no significant difference in the ES outcome between studies that prohibited and studies that allowed concurrent medication use.

Third, Sharp et al. (16) note that a problem of most comparative studies is the lack of control for therapist contact. This study too suffers from this alleged problem because patients receiving CBT spent more time with their CBT therapist when compared with the time that patients receiving SSRI spent with their pharmacotherapist. However, contact time is a characteristic inherent to the different treatment modalities and correcting this 'problem' would have severely limited the generalizability of our results.

In summary, this study demonstrated that, consistent with previous findings reported by Barlow et al. (8), a combined treatment is superior to mono-treatment although the difference between SSRI-only and the combined treatment was only modest. Also, this multicenter study supports the transportability of CBT, SSRI and CBT + SSRI from research to non-research settings. Finally, patients with moderate or severe AG were not excluded and results are thus generalizable to the whole AG continuum.

Probably the most urgent matter raised by present findings is the question of maintenance of treatment gains. The risk of relapse after tapering medication is considerable as is demonstrated in different studies in which only pharmacotherapy was studied (38). One may reason that adding CBT to the pharmacotherapy might prevent relapse. However, there are also indications that the combined treatment does not prevent relapse but even encourages it $(8,39)$. Thus, although the present findings suggest that the combined treatment has a greater effectiveness after 9 months of treatment when compared with CBT-only and, to a lesser extent, SSRI-only, the true challenge for the combined treatment may be still ahead, when the SSRIs are tapered off.

\section{Acknowledgements}

The research was granted by The Dutch Health Insurance Board (OG00-029). We thank the following treatment centers (all in the Netherlands) for their participation in the study: Ambulatorium Klinische Psychologie Groningen, Rijksuniversiteit Groningen; VU Medisch Centrum/GGZBuitenamstel, Amsterdam; GGZ-Drenthe, Assen; GGZ-Friesland, Leeuwarden; GGZ-Meerkanten, Harderwijk; Mediant GGZ Twente, Enschede; Sint Lucas Andreas Ziekenhuis, Amsterdam; GGZ Nijmegen, Nijmegen; Ambulatorium ACSW, Nijmegen; Stichting Adhesie, Almelo. The authors thank Marieke E. Timmerman for her help with the statistical analyses; Laura van Bergen for her assistance in data management; and all research assistants, CBT therapists, pharmacotherapists and patients who participated in the study.

\section{References}

1. Kessler RC, Chium WT, Jin R, Ruscio AM, Shear MK, Walters EE. The epidemiology of panic attacks, panic disorder, and agoraphobia in the national comborbidity survey replication. Arch Gen Psychiatry 2006;63:415424.

2. Ballenger JC, Davidson JRT, Lecrubier Y et al. Consensus statement on panic disorder from the international consensus group on depression and anxiety. J Clin Psychiatry 1998;59(Suppl. 8):47-54.

3. Butler aC, Chapman Je, Forman EM, Beck AT. The empirical status of cognitive-behavioral therapy: a review of meta-analyses. Clin Psychol Rev 2006;26:17-31.

4. Den Boer JA, Ter Horst GJ, Cremers tifH, Bosker FJ. Therapeutic armamentarium in anxiety disorders. In: D'Haenen H, Den Boer JA, Willner P, eds. Biological psychiatry. London: John Wiley \& Sons, 2002:1039-1062.

5. Recker Rayburn N, Otto MW. Cognitive-behavioral therapy for panic disorder: a review of treatment elements, strategies, and outcomes. CNS Spectr 2003;8:356-362.

6. Craske MG. An integrated treatment approach to panic disorder. Bull Menninger Clin 1996;60(2 (Suppl. A)):A87A104.

7. Отто MW, Smits JAJ, Reese HE. Cognitive-behavioral therapy for the treatment of anxiety disorders. J Clin Psychiatry 2004;65(Suppl. 5):34-41.

8. Barlow DH, Gorman JM, Shear MK, Woods SW. Cognitivebehavioral therapy, imipramine, or their combination for panic disorder. JAMA 2000;283:2529-2536.

9. De Beurs E, Van Balkom AJlM, Lange A, Koele P, Van Dyck R. Treatment of panic disorder with agoraphobia: comparison of fluvoxamine, placebo, and psychological panic management combined with exposure and of exposure in vivo alone. Am J Psychiatry 1995;152:683-691.

10. Fahy TJ, O’rourke D, Brophy JSW, Sciascia S. Galway study of panic disorder I: clomipramine and lofepramine in DSM-III-R panic disorder: a placebo-controlled trial. J Affect Disord 1992;25:63-76.

11. Fava Ga, Savron G, Zielezny M, Grandi S, Rafanelli C, Conti S. Overcoming resistance to exposure in panic disorder with agoraphobia. Acta Psychiatr Scand 1997;95:306-312. 
12. Johnston DG, Troyer IE, Whitsett SF. Clomipramine treatment and behaviour therapy with agoraphobic women. Can J Psychiatry 1995;40:192-199.

13. Marks IM, Gray S, Cohen D et al. Imipramine and brief therapist-aided exposure in agoraphobics having selfexposure homework. Arch Gen Psychiatry 1983;40:153162.

14. Mavissakalian M, Michelson L. Agoraphobia: relative and combined effectiveness of therapist-assisted in vivo exposure and imipramine. J Clin Psychiatry 1986;47:117-122.

15. Oehrberg S, Christiansen PE, Behnke K et al. Paroxetine in the treatment of panic disorder: a randomized, doubleblind, placebo-controlled study. $\mathrm{Br} \mathrm{J}$ Psychiatry 1995;167:374-379.

16. Sharp DM, Power KG, Simpsom RJ et al. Fluvoxamine, placebo, and cognitive behaviour therapy used alone and in combination in the treatment of panic disorder and agoraphobia. J Anxiety Disord 1996;10:219-242.

17. Telch MJ, Agras WS, Taylor CB, Roth WT, Gallen CC. Combined pharmacological and behavioral treatment for agoraphobia. Behav Res Ther 1985;23:325-335.

18. Bakker A, Van Dyck R, Spinhoven P, Van Balkom AJLM. Paroxetine, clomipramine, and cognitive therapy in the treatment of panic disorder. J Clin Psychiatry 1999;60:831838.

19. Black DW, Wesner R, Bowers W, Gabel J. A comparison of fluvoxamine, cognitive therapy, and placebo in the treatment of panic disorder. Arch Gen Psychiatry 1993;50:44-50.

20. Clark DM, Salkovskis PM, Hackmann A, Middleton H, Anastasiades P, Gelder M. A comparison of cognitive therapy, applied relaxation and imipramine in the treatment of panic disorder. Br J Psychiatry 1994;164:759-769.

21. Van Den Hout MA, Arntz A, Hoekstra R. Exposure reduced agoraphobia but not panic, and cognitive therapy reduced panic but not agoraphobia. Behav Res Ther 1994;32:447-451.

22. Sheenan DV, Lecrubier Y, Sheenan KH et al. The MiniInternational Neuropsychiatric Interview (M.I.N.I.): the development and validation of a structured diagnostic psychiatric interview for DSM-IV and ICD-10. J Clin Psychiatry 1998;59(Suppl. 20):22-33.

23. Clark DM. A cognitive approach to panic. Behav Res Ther 1986;24:461-470.

24. Craske MG, Barlow DH. Panic disorder and agoraphobia. In: BARLow DH, ed. Clinical handbook of psychological disorders. New York: Guilford, 1993.
25. Rowe MK, Craske MG. Effects of an expanding-spaced vs. massed exposure schedule on fear reduction and return of fear. Behav Res Ther 1998;36:701-717.

26. Van Balkom AJLM, Van Dyck R, Van Megen HJGM, Timmerman L, Van Vliet IM, Westenberg HGM, Witte YC, eds. Richtlijn farmacotherapie angststoornissen. Amsterdam: Dries van Ingen, Uitgeverij Boom, 1998:7-37.

27. National Institute of Mental Health. Clinical global impression. In: GuY E, ed. ECDUE assessment manual for psychopharmacology. Rockville, MD: NIMH, 1976:217222.

28. Hamilton M. The assessment of anxiety states by rating. Br J of Med Psychol 1959;32:50-55.

29. Hamilton M. A rating scale for depression. J Neurol Neurosurg Psychiatry 1960;23:56-62.

30. Marks IM, Mathews AM. Brief standard self-rating scale for phobic patients. Behav Res Ther 1979;17:263-267.

31. Arrindel WA, Ettema JHM. SCL-90: handleiding bij een multidimensionele psychopathologie-indicator. Lisse: Swets \& Zeitlinger, 2003.

32. Derogatis LR, Lipman RS, Covi L. SCL-90: an outpatient psychiatric rating scale - preliminary report. Psychopharmacol Bull 1973;9:13-28.

33. Agresti A, Caffo B. Simple and effective confidence intervals for proportions and differences of proportions result from adding two successes and two failures. Am Stat 2000;54:280-288.

34. HoLm S. A simple sequentially rejective multiple test procedure. Scand J Stat 1979;6:65-70.

35. Cohen J. A power primer. Psychol Bull 1992;112:155-159.

36. Zohar J, Westenberg HG. Anxiety disorders: a review of tricyclic antidepressants and selective serotonin reuptake inhibitors. Acta Psychiatr Scand Suppl 2000;403:39-49.

37. Отто MW, Gould RA, Mclean RYS. The effectiveness of cognitive-behavior therapy for panic disorder without concurrent medication treatment: a reply to Power and Sharp. J Psychopharmacol 1996;10:254-256.

38. Pollack MH. The pharmacotherapy of panic disorder. J Clin Psychiatry 2005;66(Suppl. 4):23-27.

39. Отto MW, Smits JAJ, ReEse HE. Combined psychotherapy and pharmacotherapy for mood and anxiety disorders in adults: Review and analysis. Clin Psychol Sci Pract 2005;12:72-86. 